\title{
Improved preparation of C-aryl glucoside SGLT2 inhibitors
}

\author{
Yong-Hai Liu, Da-Li Li*, and Lu-De Lu \\ School of Chemical Engineering, Nanjing University of Science and Technology, Nanjing 210094, \\ P. R. China \\ E-mail:dreamlyh@sohu.com
}

\begin{abstract}
A novel approach for the preparation of $(2 \mathrm{~S}, 3 \mathrm{R}, 4 \mathrm{R}, 5 \mathrm{~S}, 6 \mathrm{R})-2-(3-(4-e t h y l b e n z y 1)-4-c h l o r o-$ phenyl)-6-(hydroxymethyl)-tetrahydro-2H-pyran-3,4,5-triol 1 is developed. The target compound via six steps is synthesized from 5-bromo-2-chlorobenzoic acid and the isomers of undesired ortho-products were avoided during the preparation.
\end{abstract}

Keywords: Type 2 diabetes, sodium glucose co-transporter 2, inhibitor, preparation

\section{Introduction}

Sodium glucose co-transporter 2 (SGLT2) plays a key role in maintaining glucose equilibrium in the human body. ${ }^{1}$ Much attention has been given to SGLT2 as a molecular target to directly induce glucose excretion and to safely normalize plasma glucose in the treatment of type 2 diabetes. ${ }^{2}$ The reformative subset of SGLT2 inhibitors to be explored was the carbon glycosides in which the bond between the glucose and aglycone is a carbon-carbon bond. ${ }^{3,4}$ Recently, it was reported that (2S,3R,4R,5S,6R)-2-(3-(4-ethylbenzyl)-4-chlorophenyl)-6-(hydroxymethyl)-

tetrahydro-2H-pyran-3,4,5-triol 1 may be advancing to clinical development to directly induce glucose excretion and to safely normalize plasma glucose in the treatment of type 2 diabetes. The reported synthetic route of $\mathbf{1}$ was shown in Scheme 1., 6 

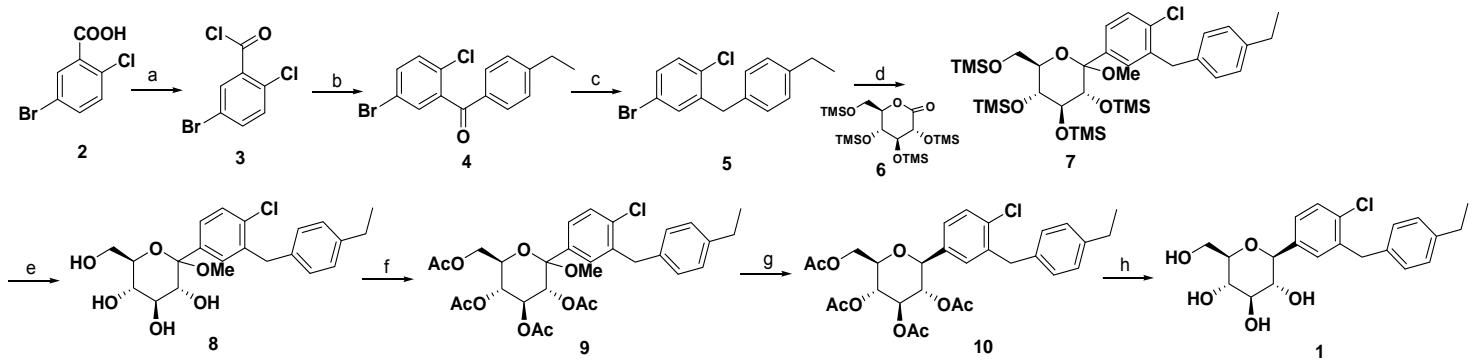

Scheme 1. Reagents and conditions: (a) $\mathrm{SOCl}_{2}$, dichloromethane (DCM), r.t.; (b) ethylbenzene, $\mathrm{AlCl}_{3},-15^{\circ} \mathrm{C}$; (c) $\mathrm{Et}_{3} \mathrm{SiH}, \mathrm{TFA}, \mathrm{CF}_{3} \mathrm{SO}_{3} \mathrm{H}$, reflux; (d) THF, toluene, $n$ - BuLi, $-55^{\circ} \mathrm{C}$; (e) $\mathrm{CH}_{3} \mathrm{OH}$, $\mathrm{CH}_{3} \mathrm{SO}_{3} \mathrm{H}, 20^{\circ} \mathrm{C}$; (f) diiso-propylethylamine, DMAP, $\mathrm{Ac}_{2} \mathrm{O}, 20^{\circ} \mathrm{C}$; (g)DCM, $\mathrm{Et}_{3} \mathrm{SiH}_{2} \mathrm{BF}_{3} . \mathrm{Et}_{2} \mathrm{O}$, $-20^{\circ} \mathrm{C}$; (h) $\mathrm{LiOH}, \mathrm{CH}_{3} \mathrm{OH}, 20^{\circ} \mathrm{C}$.

\section{Results and Discussion}

The important intermediate $\mathbf{4}$ was prepared by Friedel-Crafts reaction but the selectivity of the ortho-position and para-position of the reaction was unsolved according to the procedure of William et al. ${ }^{6}$ In addition, compound $\mathbf{7}$ was synthesized by coupling reaction between $\mathbf{5}$ and $\mathbf{6}$ in unsatisfactory yield and the synthetic route needed eight steps which limited a large scale production. ${ }^{6}$

In this report a novel synthetic route was employed to prepare $\mathbf{4}$ by the reactions of Weinreb and Grignard at the first and second steps which the isomers of undesired ortho-products were avoided (Scheme 2). Then 5 was transformed to D-glucopyranoside 12 in excellent yield. Treatment of 12 with $\mathrm{Et}_{3} \mathrm{SiH}$ and $\mathrm{BF}_{3}$. $\mathrm{Et}_{2} \mathrm{O}$ in DCM for $5 \mathrm{~h}$ afforded the compound 13, which was hydrogenated under $0.1 \mathrm{MPa}$ hydrogen at room temperature to produce 1 in about $70.7 \%$ overall yield. The structure of the target compound was confirmed by elemental analysis, ${ }^{1} \mathrm{H} N M R,{ }^{13} \mathrm{C}$ NMR and MS.

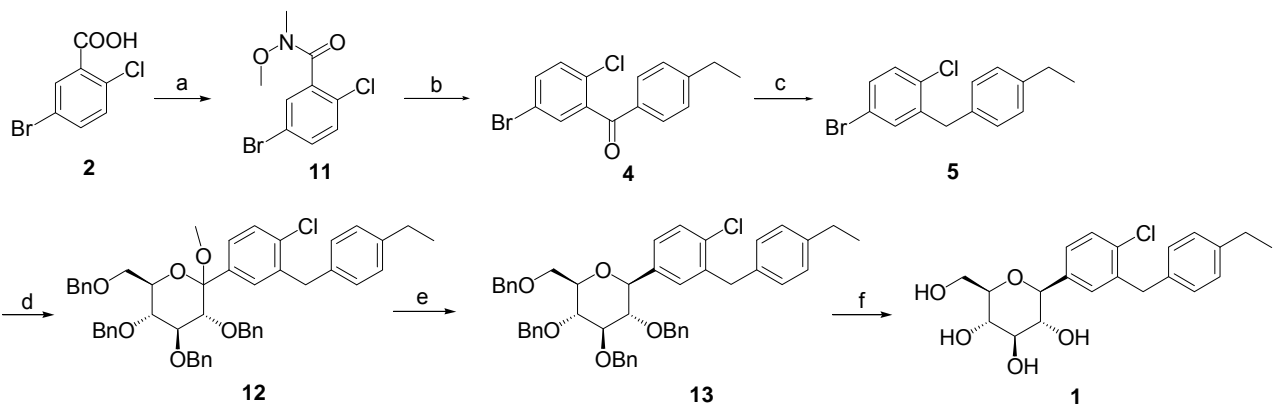

Scheme 2. Reagents and conditions: (a) DMF/CDI/Et ${ }_{3} \mathrm{~N} / \mathrm{N}$-methoxymethanamine hydrochloride, $30^{\circ} \mathrm{C}$; (b) $\mathrm{THF} /\left(4\right.$-ethylphenyl)magnesium bromide, $30^{\circ} \mathrm{C}$; (c) $\mathrm{Et} 33 \mathrm{SiH} / \mathrm{TFA} / \mathrm{CF}_{3} \mathrm{SO}_{3} \mathrm{H}$, reflux; (d) 
(1) THF/toluene, $n$-BuLi ,-55 ${ }^{\circ} \mathrm{C}$; (2) (3R,4S,5R,6R)-3,4,5-tris(benzyloxy)-6- (benzyloxymethyl)tetrah- ydro-pyran-2-one, $-78^{\circ} \mathrm{C}$; (e) $\mathrm{DCM} / \mathrm{CH}_{3} \mathrm{CN} / \mathrm{Et}_{3} \mathrm{SiH}_{2} \mathrm{BF}_{3} \cdot \mathrm{Et}_{2} \mathrm{O}$, below $0^{\circ} \mathrm{C}$; (f) $\mathrm{EA} / \mathrm{MeOH} / \mathrm{H}_{2} ; 0.1 \mathrm{MPa}, 25^{\circ} \mathrm{C}$.

In order to demonstrate the influence of solvent, a series of solvents were used in this protocol for deprotection of $\mathbf{1 3}$ and the results are shown in Table 1. A mixture of $\mathbf{1}$ and 1a were found in benzene, toluene, chlorobenzene and 1,2-dichlorobenzene during the deprotection reaction. However, when 1,2-dichlorobenzene was added (20 equimolar amounts) to the reaction system, by-product 1a could not be detected by LC-MS (Table 1, Entry 4). Mechanically, the possible reason is in the existence of 1,2-dichlorobenzene in this protocol, its chlorine group can be reduced at first and the resultant hydrochloride can gradually inhibit reduction of the chlorine group of 1.

Table 1. Synthesis of compound 1 by deprotection of benzyl group

\begin{tabular}{|c|c|c|c|c|}
\hline Entry & Solvent & $\begin{array}{l}\text { Equimolar } \\
\text { amounts }\end{array}$ & $\begin{array}{c}\text { Compound } 1 \\
\text { Purity }(\%)^{\alpha}\end{array}$ & $\begin{array}{c}\text { By-product 1a } \\
\text { Purity }(\%)^{\alpha}\end{array}$ \\
\hline \multirow{2}{*}{1} & \multirow{2}{*}{ Benzene } & 10 & 41.50 & 58.00 \\
\hline & & 20 & 40.50 & 58.50 \\
\hline \multirow{2}{*}{2} & \multirow{2}{*}{ Toluene } & 10 & 41.00 & 58.60 \\
\hline & & 20 & 40.30 & 58.40 \\
\hline \multirow{2}{*}{3} & \multirow{2}{*}{ Chlorobenzene } & 10 & 45.05 & 54.05 \\
\hline & & 20 & 49.40 & 49.50 \\
\hline \multirow{2}{*}{4} & \multirow{2}{*}{ 1,2-Dichlorobenzene } & 10 & 87.32 & 10.32 \\
\hline & & 20 & 99.03 & 0 \\
\hline
\end{tabular}

${ }^{\alpha}$ Determined on HPLC analysis of crude products before purification.

In conclusion, a more novel, and effective approach for the synthesis $\mathbf{1}$ in about $71 \%$ overall yield was found. The undesired ortho-products were avoided for the preparation of intermediate 4 and two synthetic steps were reduced.

\section{Experimental Section}

General Procedures. All the reagents were obtained from suppliers and were not purified. Melting points were measured on a PHMK179-2454 apparatus. Elemental analysis (C, H, N) was 
determined with a Perkin-Elmer 240c instrument, their results were found to be in good agreement $( \pm 0.3 \%)$ with the calculated values. ${ }^{1} \mathrm{H}$ NMR was measured on a Bruker AM-400MHz spectro-meter, ${ }^{13} \mathrm{CNMR}$ was measured on a Bruker AM-300MHz spectro-meter. EI mass spectral measurement was carried out on a Waters alliance 2695 with acetonitrile and water as a mobile phase.

The 5 was synthesized according to Ref. 6. The 4, 5, are known compounds, which were characterized by Elemental analysis, ${ }^{1} \mathrm{H}$ NMR, EI mass spectral data compared with literature data reports. ${ }^{6}$

5-Bromo-2-chloro- $\boldsymbol{N}$-methoxy- $\boldsymbol{N}$-methylbenzamide (11). To a solution of 5-bromo-2-chlorobenzoic acid (23.5 g, $100 \mathrm{mmol})$ in $100 \mathrm{~mL}$ anhydrous DMF , $N, N^{\prime}$-Carbonyldiimidazole (CDI) $(18.3 \mathrm{~g}, 130 \mathrm{mmol})$ was added in portions. After all of the CDI was added, $\mathrm{Et}_{3} \mathrm{~N}(13.1 \mathrm{~g}, 130 \mathrm{mmol})$ was added to the mixture, stirred at $25^{\circ} \mathrm{C}$ for $1 \mathrm{~h}$, then $N$-methoxymethanamine hydrochloride $(12.7 \mathrm{~g}, 130 \mathrm{mmol})$ was added, the reaction mixture was stirred at $25^{\circ} \mathrm{C}$ for $10 \mathrm{~h}$, then the mixture was poured into $1000 \mathrm{~mL}$ water and extracted with ether $(1000 \mathrm{~mL})$, the organic phase was separated and washed with water, brine and dried with anhydrous $\mathrm{Na}_{2} \mathrm{SO}_{4}$, then was concentrated in vacuo to give a white solid $(27.80 \mathrm{~g}, 99.8 \%)$. mp: 78-80 ${ }^{\circ} \mathrm{C} .{ }^{1} \mathrm{H}$ NMR $\left(400 \mathrm{MHz}, \mathrm{CDCl}_{3}\right): \delta=7.474(\mathrm{~m}, 2 \mathrm{H}), 7.286(\mathrm{~d}, 1 \mathrm{H}, J=8.0 \mathrm{~Hz}), 3.572(\mathrm{~s}, 3 \mathrm{H})$, $3.345(\mathrm{~s}, 3 \mathrm{H}) ;{ }^{13} \mathrm{C} \mathrm{NMR}\left(300 \mathrm{MHz}, \mathrm{CDCl}_{3}\right): \delta=167.05,137.32,133.59,131.38,130.85,129.88$, 120.48, 61.85, 32.63; MS [EI]: $m / z 280\left[\mathrm{M}^{+}\right]$. Anal. Calcd for $\mathrm{C}_{9} \mathrm{H}_{9} \mathrm{BrClNO}_{2}$ : C 38.81, H 3.26, N 5.03; Found C 38.76, H 3.27, N 5.01.

(5-Bromo-2-chlorophenyl)(4-ethylphenyl)methanone (4). To a solution of 11 (13.9 g, $50 \mathrm{mmol})$ in anhydrous THF (100 mL), (4-ethylphenyl)magnesium bromide (1 M in THF, $60 \mathrm{~mL})$ was added dropwise to the reactor below $30^{\circ} \mathrm{C}$. The reaction mixture was stirred at $25^{\circ} \mathrm{C}$ for $2 \mathrm{~h}$, then the reaction was quenched with saturated $\mathrm{NH}_{4} \mathrm{Cl}$, extracted with ethyl acetate (EA), washed with water, brine and dried with anhydrous $\mathrm{Na}_{2} \mathrm{SO}_{4}$, and concentrated in vacuo to give an oil (16.89 g, 98.2\%). ${ }^{1} \mathrm{H}$ NMR (400 MHz, $\left.\mathrm{CDCl}_{3}\right) \delta=7.73(\mathrm{~d}, 2 \mathrm{H}, J=8.0 \mathrm{~Hz}$ ), $7.54(\mathrm{dd}, 1 \mathrm{H}, J=2.4 \mathrm{~Hz}, J=8.8$ $\mathrm{Hz}), 7.49$ (d, 1H, $J=2.4 \mathrm{~Hz}), 7.33$ (d, 2H, $J=8.4 \mathrm{~Hz}$ ), 7.29 (s, 1H ), 2.73 (q, 2H, $J=7.6), 1.272$ (t, $3 \mathrm{H}, J=7.6 \mathrm{~Hz}) ;{ }^{13} \mathrm{C} \mathrm{NMR}\left(300 \mathrm{MHz}, \mathrm{CDCl}_{3}\right): \delta=193.0,149.2,141.0,136.0,133.7,134.3,132.3$,

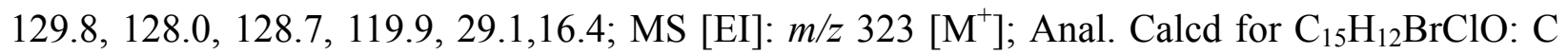
55.67, H 3.74; Found C 55.61, H 3.75.

2-(4-Ethylbenzyl)-4-bromo-1-chlorobenzene (5). ${ }^{1} \mathrm{H} \mathrm{NMR}\left(400 \mathrm{MHz}, \mathrm{CDCl}_{3}\right) \delta=7.24-7.31$ (m, $3 \mathrm{H}), 7.176(\mathrm{~d}, 2 \mathrm{H}, J=8.0 \mathrm{~Hz}), 7.13(\mathrm{~d}, 2 \mathrm{H}, J=8.4 \mathrm{~Hz}), 4.055$ (s, 2H ), 2.664 (q, 2H, $J=7.6 \mathrm{~Hz})$, 1.267 (t, 3H, $J=7.6 \mathrm{~Hz}) ;{ }^{13} \mathrm{C} \mathrm{NMR}\left(300 \mathrm{MHz}, \mathrm{CDCl}_{3}\right): \delta=142.9,141.6,136.2,134.2,133.7,131.4$, 131.1, 129.4, 128.6, 121.0, 39.2, 29.0, 16.1; MS [EI]: $m / z 309\left[\mathrm{M}^{+}\right]$; Anal. Calcd for $\mathrm{C}_{15} \mathrm{H}_{14} \mathrm{BrCl}$ : C 58.19, H 4.56; Found C 58.11, H 4.57.

(3R,4S,5R,6R)-2-(3-(4-Ethylbenzyl)-4-chlorophenyl)-3,4,5-tris(benzyloxy)-6-(benzyloxymet hyl)-2-methoxy-tetrahydro-2H-pyran (12). To a stirred $-78^{\circ} \mathrm{C}$ solution of 5 (3.03 g, $\left.9.8 \mathrm{mmol}\right)$ in $15 \mathrm{~mL}$ of $1: 2$ anhydrous THF/toluene was slowly added $2.5 \mathrm{M} \mathrm{n}$-BuLi (4.4 mL, $11 \mathrm{mmol})$ in hexane to maintain the temperature below $-55^{\circ} \mathrm{C}$. 
After stirring for $10 \mathrm{~min}$, this solution was transferred to a stirred $-78^{\circ} \mathrm{C}$ solution of (3R,4S,5R,6R)-3,4,5-tris(benzyloxy)-6-(benzyloxymethyl)-tetrahydro-pyran-2-one (5.28g, $9.8 \mathrm{mmol})$ in toluene $(15 \mathrm{~mL})$ below $-55^{\circ} \mathrm{C}$. The solution was stirred for $30 \mathrm{~min}$ at $-78^{\circ} \mathrm{C}$, quenched by addition of $10 \mathrm{~mL}$ of $\mathrm{MeOH}$ containing methanesulfonic acid $(0.7 \mathrm{~mL})$, the mixture was stirred at $20^{\circ} \mathrm{C}$ overnight. The reaction was quenched with saturated $\mathrm{NaHCO}_{3}$ and extracted with EA. The organic phase was separated and washed with brine, dried (anhydrous $\mathrm{Na}_{2} \mathrm{SO}_{4}$ ) and concentrated in vacuo to give an oil, crystallization from EtOH $(14 \mathrm{~mL})$ to get a light brown crystals $\quad 6.22 \mathrm{~g}, 81 \%$. mp: $97-99^{\circ} \mathrm{C} ;{ }^{1} \mathrm{H} \mathrm{NMR}\left(400 \mathrm{MHz}, \mathrm{CDCl}_{3}\right): \delta=7.49$ (d, $\left.1 \mathrm{H}, J=1.5 \mathrm{~Hz}\right)$, $7.35 \sim 7.40(\mathrm{~m}, 6 \mathrm{H}), 30 \sim 7.35(\mathrm{~m}, 9 \mathrm{H}), 7.21 \sim 7.27(\mathrm{~m}, 5 \mathrm{H}), 7.08(\mathrm{~d}, 4 \mathrm{H}, J=1.2 \mathrm{~Hz}), 7.04(\mathrm{~d}, 2 \mathrm{H}$, $J=8.4 \mathrm{~Hz}), 4.89 \sim 4.97(\mathrm{~m}, 3 \mathrm{H}), 4.59 \sim 4.69(\mathrm{~m}, 3 \mathrm{H}), 4.51$ (d, 1H, $J=10.8 \mathrm{~Hz}), 4.14 \sim 4.21(\mathrm{~m}, 2 \mathrm{H})$, $3.98(\mathrm{~d}, 1 \mathrm{H}, J=8.4 \mathrm{~Hz}), 3.94$ (s, 1H), $3.77 \sim 3.87$ (m, 4H), 3.36 (d, 1H, J=9.6Hz), $3.11(\mathrm{~s}, 3 \mathrm{H}), 2.61$ (q, $2 \mathrm{H}, J=7.6 \mathrm{~Hz}), 1.22$ (t, $3 \mathrm{H}, J=7.6 \mathrm{~Hz}) ;{ }^{13} \mathrm{C} \mathrm{NMR}\left(300 \mathrm{MHz}, \mathrm{CD}_{3} \mathrm{OD}\right): \delta=139.0,138.8,137.7$, 130.9 , 129.7, 129.1, 129.0, 128.9, 128.8, 128.6, 128.4, 128.3, 128.2, 128.1, 128.0, 127.4, 101.5, $85.8,83.9,78.8,77.9,77.5,76.5,76.2,75.6,73.9,72.6,69.2,49.6,39.4,28.8,16.0 ; \mathrm{MS}[\mathrm{EI}]: \mathrm{m} / z$ $806\left[\mathrm{M}^{+}+\mathrm{Na}^{+}\right] ; 801\left[\mathrm{M}^{+}+\mathrm{H}_{2} \mathrm{O}\right] ; 752\left[\mathrm{M}^{+}-\mathrm{OMe}\right]$; Anal. Calcd for $\mathrm{C}_{50} \mathrm{H}_{51} \mathrm{ClO}_{6}: \mathrm{C}$ 76.66, H 6.56; Found C 76.57, H 6.57.

(2S,3S,4R,5R,6R)-2-(3-(4-ethylbenzyl)-4-chlorophenyl)-3,4,5-tris(benzyloxy)-6-(benzyloxym ethyl)-tetrahydro-2H-pyran (13). A stirred solution of 12 (3.92 g, $5 \mathrm{mmol})$ in $20 \mathrm{~mL}$ solution $\left(\mathrm{DCM} / \mathrm{CH}_{3} \mathrm{CN}=1: 1 ; \mathrm{v} / \mathrm{v}\right)$ containing $\mathrm{Et}_{3} \mathrm{SiH}(1.16 \mathrm{~g}, 9.97 \mathrm{mmol})$ was cooled to $-20^{\circ} \mathrm{C}$. Prior to addition of $\mathrm{BF}_{3} \cdot \mathrm{Et}_{2} \mathrm{O}(1.1 \mathrm{~g}, 7.7 \mathrm{mmol})$ below $0^{\circ} \mathrm{C}$, and then, the reaction was stirred for $5 \mathrm{~h}$ below $25^{\circ} \mathrm{C}$. The saturated solution of $\mathrm{NaHCO}_{3}$ was added until $\mathrm{pH}$ to above 7 . The organic phase was separated, and the water phase was extracted with $3 \times 60 \mathrm{~mL}$ DCM, the combined organic phase was washed with saturated $\mathrm{NaHCO}_{3}$, dried with anhydrous $\mathrm{Na}_{2} \mathrm{SO}_{4}$, and concentrated in vacuo to give an oil. $3.57 \mathrm{~g}, 95 \%$. ${ }^{1} \mathrm{H}$ NMR $\left(400 \mathrm{MHz}, \mathrm{CDCl}_{3}\right): \delta=7.41(\mathrm{~m}, 1 \mathrm{H}), 7.27 \sim 7.37(\mathrm{~m}, 16 \mathrm{H})$, $7.20 \sim 7.26(\mathrm{~m}, 4 \mathrm{H}), 7.05 \sim 7.15(\mathrm{~m}, 4 \mathrm{H}), 6.93(\mathrm{~d}, 2 \mathrm{H}, J=8.4 \mathrm{~Hz}), 4.77 \sim 4.97(\mathrm{~m}, 3 \mathrm{H}), 4.55 \sim 4.69(\mathrm{~m}$, $3 \mathrm{H}), 4.40 \sim 4.51(\mathrm{~m}, 1 \mathrm{H}), 4.04 \sim 4.21(\mathrm{~m}, 4 \mathrm{H}), 3.75 \sim 3.89(\mathrm{~m}, 4 \mathrm{H}), 3.42 \sim 3.62(\mathrm{~m}, 2 \mathrm{H}), 2.61(\mathrm{q}, 2 \mathrm{H}$, $J=7.6 \mathrm{~Hz}), 1.22(\mathrm{t}, 3 \mathrm{H}, J=7.6 \mathrm{~Hz}) ;{ }^{13} \mathrm{C} \mathrm{NMR}\left(300 \mathrm{MHz}, \mathrm{CD}_{3} \mathrm{OD}\right) \delta=142.0,138.6,138.2,138.1$, $137.5,136.5,130.6,129.5,129.0,128.7,128.4,128.3,128.2,128.0,127.7,126.7,86.7,84.1,80.9$, 79.4, 78.3, 77.4, 76.6, 75.7, 75.1, 75.0, 73.4, 69.0, 38.8, 28.4, 15.5; MS [EI]: $m / z 776\left[\mathrm{M}^{+}+\mathrm{Na}^{+}\right]$; $771\left[\mathrm{M}^{+}+\mathrm{H}_{2} \mathrm{O}\right] ; 753\left[\mathrm{M}^{+}\right]$; Anal. Calcd for $\mathrm{C}_{49} \mathrm{H}_{49} \mathrm{ClO}_{5}$ : C 78.12, H 6.56; Found C 78.05, H 6.57 (2S,3R,4R,5S,6R)-2-(3-(4-Ethylbenzyl)-4-chlorophenyl)-6-(hydroxymethyl)-tetrahydro-2Hpyran-3,4,5-triol (1). To a solution of $13(2.51 \mathrm{~g}, 3.33 \mathrm{mmol})$ in $2: 3 \mathrm{EA} / \mathrm{MeOH}(30 \mathrm{~mL}), 0.25 \mathrm{~g}$ Palladium on carbon and $10 \mathrm{~mL} 1,2$-Dichlorobenzene was added in turn. The air of reactor is removed by argon, then the $0.1 \mathrm{MPa} \mathrm{H}_{2}$ was added for $12 \mathrm{~h}$ at $25^{\circ} \mathrm{C}$. The solvent was filtrated, filter cake was washed by EA, the filtrate was concentrated in vacuo to give an oil. The oil was purified with silicon column to get a glassy off white solid $(1.24 \mathrm{~g}, 95 \%)$. mp: $46-48^{\circ} \mathrm{C}$, Yield $96 \%$, ${ }^{1} \mathrm{H}$ NMR (400 MHz, CD $\left.{ }_{3} \mathrm{OD}, \delta \mathrm{ppm}\right): 7.34(\mathrm{~m}, 2 \mathrm{H}), 7.263(\mathrm{dd}, 1 \mathrm{H}, J=2.0 \mathrm{~Hz}, J=8.0 \mathrm{~Hz}), 7.08$ (m, $4 \mathrm{H}), 4.056(\mathrm{~m}, 3 \mathrm{H}), 3.86(\mathrm{~d}, 1 \mathrm{H}, J=12.4 \mathrm{~Hz}), 3.67(\mathrm{~m}, 1 \mathrm{H}), 3.37-3.46(\mathrm{~m}, 3 \mathrm{H}), 3.264(\mathrm{~d}, 1 \mathrm{H}, J=8.8$ $\mathrm{Hz}), 2.578$ (q, 2H, $J=7.6 \mathrm{~Hz}), 1.186(\mathrm{t}, 3 \mathrm{H}, J=7.6 \mathrm{~Hz}) ;{ }^{13} \mathrm{C} \mathrm{NMR}\left(300 \mathrm{MHz}, \mathrm{CD}_{3} \mathrm{OD}, \delta \mathrm{ppm}\right)$ : $143.2,140.0,139.7,138.1,134.5,131.98,130.1,129.8,128.8,128.2,82.8,82.14,79.7,76.4,71.9$, 
63.1, 39.7, 29.4, 16.25; MS [ESI]: $m / z 393\left[\mathrm{M}^{+}\right] ; 410\left[\mathrm{M}^{+}+\mathrm{H}_{2} \mathrm{O}\right] ; 7862\left[\mathrm{M}^{+}\right]$; Anal. Calcd for $\mathrm{C}_{21} \mathrm{H}_{25} \mathrm{ClO}_{5}$ : C 64.20, H 6.41; Found C 64.11, H 6.43.

\section{References}

1. (a) Mathis, D.; Vence, L.; Benoist, C. Nature 2001, 414, 792. (b) Kaiser, N.; Leibowitz, G.; Nesher, R. J. Pediatric Endocrinol. Metab. 2003, 16, 5. (c) Cerasi, E.; Kaiser, N.; Leibowitz, G. Diabetes Metab. 2000, 26, 13

2. (a) Wright, E. M.; Turk, E. Eur. J. Physiol. 2004, 447, 510. (b) Scheepers, A.; Joost, H.; Schurmann, A. J. Parenteral. Enteral. Nutrition 2004, 28, 365. (c) Asano, T.; Anai, M.; Sakoda, H. Drugs Fut. 2004, 29, 461. (d) Asano, T.; Ogihara, T.; Katagiri, H. Curr. Med. Chem. 2004, 11, 2717

3. (a) Ehrenkranz, R. R. L.; Lewis, N. G.; Kahn, C.; Roth, J. Diabetes Metab. Res. Rev. 2005, 21, 31. (b) Wells, R.; Pajor, A.; Kanai, Y. Am. J. Physiol. 1992, 263, 459. (c) Kanai, Y.; Lee, W.; You, G. J. Clin. Invest. 1994, 93, 397. (d) Oku, A.; Ueta, K.; Arakawa, K. Diabetes 1999, 48, 1794

4. Anthony, L. Expert Opin. Ther. Patents 2005, 15, 1531

5. Matthias, E.; Frank, H.; Peter, E. U.S. Pat. 2006, 0074031

6. William, W.; Wei, M. U.S. Pat. 2006, 0063722 